\title{
Head and neck radiotherapy on the MR linac: a multicenter planning challenge amongst MRIdian platform users
}

\author{
Madalyne Chamberlain ${ }^{1}$ (D) - Jerome Krayenbuehl' ${ }^{1}$ Janita E. van Timmeren' ${ }^{1}$ - Lotte Wilke $^{1}$ (D) $\cdot$ \\ Nicolaus Andratschke ${ }^{1}$ Helena Garcia Schüler ${ }^{1}$ Stephanie Tanadini-Lang ${ }^{1}$ Matthias Guckenberger ${ }^{1}$. \\ Panagiotis Balermpas ${ }^{1}$ (iD
}

Received: 12 October 2020 / Accepted: 22 March 2021 / Published online: 23 April 2021

(c) The Author(s) 2021

\begin{abstract}
Purpose Purpose of this study is to evaluate plan quality on the MRIdian (Viewray Inc., Oakwood Village, OH, USA) system for head and neck cancer (HNC) through comparison of planning approaches of several centers.

Methods A total of 14 planners using the MRIdian planning system participated in this treatment challenge, centrally organized by ViewRay, for one contoured case of oropharyngeal carcinoma with standard constraints for organs at risk (OAR). Homogeneity, conformity, sparing of OARs, and other parameters were evaluated according to The International Commission on Radiation Units and Measurements (ICRU) recommendations anonymously, and then compared between centers. Differences amongst centers were assessed by means of Wilcoxon test. Each plan had to fulfil hard constraints based on dose-volume histogram (DVH) parameters and delivery time. A plan quality metric (PQM) was evaluated. The PQM was defined as the sum of 16 submetrics characterizing different DVH goals.

Results For most dose parameters the median score of all centers was higher than the threshold that results in an ideal score. Six participants achieved the maximum number of points for the OAR dose parameters, and none had an unacceptable performance on any of the metrics. Each planner was able to achieve all the requirements except for one which exceeded delivery time. The number of segments correlated to improved PQM and inversely correlated to brainstem $\mathrm{D}_{0.1 \mathrm{cc}}$ and to Planning Target Volume1 (PTV) $\mathrm{D}_{0.1 \mathrm{cc}}$. Total planning experience inversely correlated to spinal canal dose.

Conclusion Magnetic Resonance Image (MRI) linac-based planning for HNC is already feasible with good quality. Generally, an increased number of segments and increasing planning experience are able to provide better results regarding planning quality without significantly prolonging overall treatment time.
\end{abstract}

Keywords MR-adaptive treatment planning $\cdot$ Step-and-shoot IMRT $\cdot$ MR-guided radiation therapy $\cdot$ Treatment plan comparison · Low field magnetic resonance imaging

\section{Introduction}

In recent years various advances in image-guided radiotherapy (IGRT) have led to improved precision and accuracy in radiation treatment delivery for head and neck cancer (HNC) [1-3]. Three-dimensional image guidance for patient setup allows corrections and could also reduce fail-

\footnotetext{
Availability of data and materials All of the original data are available upon request.

Madalyne Chamberlain

Madalyne.chamberlain@usz.ch

1 Department of Radiation Oncology, University Hospital Zurich, Zurich, Switzerland
}

ures caused by anatomical changes of tumor or other organs, e.g., in case of weight changes during the 6-7-week treatment $[4,5]$. Furthermore, the ultimate goal of all novel radiotherapy application techniques is reduction of toxicity, which has already been demonstrated for step-and-shoot intensity-modulated radiotherapy (IMRT) in the past [6]. The vast majority of linear accelerators (linacs) used for HNC treatment nowadays have integrated cone-beam computed tomography (CBCT) or, in the case of tomotherapy, megavoltage (MV) computed tomography (CT) for image guidance $[1,7]$. Although these CT scans can detect some anatomical changes and allow patient positioning based on radiopaque structures like bone or metal fiducials, image guidance is generally suboptimal due to low soft tissue contrast, artefacts (e.g., caused by denture or internal fixa- 


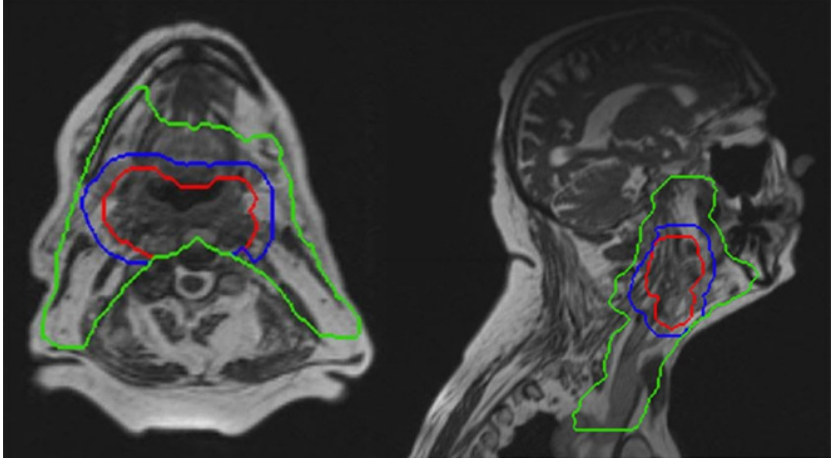

Fig. 1 Planning MRI image and PTV contours of the clinical case used in the planning challenge. Three dose levels depicted in red (70 Gy), blue (59.4 Gy), and green (54Gy). MRI magnetic resonance imaging, $P T V$ planning target volume

tion plates), and increased noise level. Furthermore, x-raybased IGRT leads to additional dose exposure in this sensible region, leading to enhanced deterministic and stochastic risks like cataracts and secondary malignancies, especially if daily imaging is needed [8]. Hybrid platforms consisting of a linac with integrated magnetic resonance imaging (MRI) have gained approval for clinical use only in the last few years, allowing superior soft tissue contrast and live imaging during beam application, without additional exposure to ionizing radiation [9]. These features could lead to the future establishment of MR linacs as the optimal platforms to treat diseases like HNC. In such cases, a relatively high radiation dose has to be applied with a steep gradient to low $\mathrm{x}$-ray contrast organs at risk (OARs) like salivary glands and pharyngeal constrictor muscles. For these patients both coverage of the planning target volume (PTV) and maximal sparing of OARs are crucial, as they are usually treated with full curative intent and late sequelae with impairment of life quality are a common problem after head and neck irradiation.

In this study, we evaluated the different planning approaches used amongst MRIdian (MRIdian System; ViewRay Inc. Oakwood Village, $\mathrm{OH})$ users. We aimed to investigate application features and feasibility of this approach, with the ultimate goal to improve our plan quality and consequently implement the benefits of MR linac technology in standard routine for the majority of HNC cases in the near future.

\section{Methods}

\section{Case selection}

A pre-contoured real-life case of a patient with $\mathrm{cT} 4 \mathrm{~b} \mathrm{cN} 1$ p16-negative squamous cell carcinoma of the posterior oropharyngeal wall was distributed to all participating cen- ters through an online platform. Contouring of high- and intermediate-risk target volumes and neck lymphatics was performed according to international recommendations [10, 11]. An approach according to the guidelines published by Gregoire et al. [11] for clinical target volume (CTV) definition and Biau et al. [10] for the selection of the lymph node target volumes to be irradiated was used. Briefly, a Gross Target Volume (GTV)-to-CTV margin of $10 \mathrm{~mm}$ cropped for anatomic boundaries (e.g., air, bone) was adopted for CTV2 (59.4Gy) and of $5 \mathrm{~mm}$ for CTV1 (69.96 Gy). The CTV-to-PTV margins amounted to $3 \mathrm{~mm}$ for all three PTVs [12]. The consensus guidelines published by Brouwer et al. [13] were used for definition of OARs. Representative images can be seen in Fig. 1. The prescription was on three dose levels with a simultaneous integrated boost (SIB) technique in 33 factions: 69.96 Gy should be applied to the high-risk volume (PTV1), 59.4 Gy to the intermediaterisk volume (PTV2), and 54 Gy to the "elective," low-risk volume (PTV3). The patient signed informed consent for data and image use (including the MRI images presented here) according to institutional standards.

The planning MRI consisted of an MRI simulation image in the treatment position performed on the MRIdian system $(0.35 \mathrm{~T})$. One imaging sequence is currently available on the MRIdian system, which is a True FISP, which gives a mixture of T2-/T1-weighted contrast. The planning MRI was a high-resolution $(1.5 \mathrm{~mm} \times 1.5 \mathrm{~mm} \times 1.5 \mathrm{~mm}$ resolution) MRI image with a field size of $50 \mathrm{~cm} \times 45 \mathrm{~cm} \times 43 \mathrm{~cm}$, which gives an acquisition time of $172 \mathrm{~s}$. A CT simulation image was also included to use for dose calculation, which was imaged with $120 \mathrm{kV}$ and 3-mm slices in the treatment position. The patient was positioned on a head coil which was placed over a head rest, then a thermoplastic mask was placed over the patient and these items, and finally, the top head coil was positioned. An example of the positioning can be viewed in Supplementary Fig. 1.

\section{Platform and planning specifications}

All of the important planning constraints were distributed to the participating centers before planning and can be found in Supplementary table 1 .

This treatment planning challenge was organized by ViewRay and held on the ProKnow (ProKnow LLC., Sanford, FL, USA; https://proknowsystems.com/) online platform. Participants had 1 month to download the dataset from the ProKnow platform and create a treatment plan for this case. All plans were created using the ViewRay treatment planning system (TPS) which uses a Monte Carlo (MC) dose calculation algorithm taking into account the magnetic field of $0.35 \mathrm{~T}$. Inverse optimization using objectives and constraints was used to create step-and-shoot IMRT plans [14]. The CT was registered to the planning 
33 fractions: 69.96 Gy to PTV1, 59.4 Gy to PTV2, 54 Gy to PTV3

\begin{tabular}{|c|c|c|c|c|c|c|c|c|c|}
\hline \multicolumn{2}{|c|}{ \# METRIC ID (19 Total Metrics) } & \multirow{2}{*}{$\begin{array}{l}\begin{array}{l}\text { WEIGHT } \\
(150)\end{array} \\
15\end{array}$} & \multicolumn{7}{|l|}{ PERFORMANCE BINS } \\
\hline & Dose (Gy) covering 95 (\%) of the PTV1 & & $\begin{array}{l}<62.6 \\
\text { UNACCEPTABLE }\end{array}$ & \multicolumn{2}{|l|}{$\begin{array}{l}62.601 \\
\text { ACCEPTABLE }\end{array}$} & \multicolumn{3}{|l|}{$\begin{array}{l}66.2 \\
\text { GOOD }\end{array}$} & $\begin{array}{l}69.96 \\
\text { IDEAL }\end{array}$ \\
\hline [02] & Dose (Gy) covering $0.1(\mathrm{cc})$ of the PTV1 & 15 & $\begin{array}{l}>80.5 \\
\text { UNACCEPTABLE }\end{array}$ & \multicolumn{2}{|l|}{$\begin{array}{l}80.5 \\
\text { MARGINAL }\end{array}$} & \multicolumn{2}{|l|}{$\begin{array}{l}77 \\
\text { ACCEPTABLE }\end{array}$} & $\begin{array}{l}75 \\
\text { GOOD }\end{array}$ & $\begin{array}{l}73.5 \\
\text { IDEAL }\end{array}$ \\
\hline [03] & Dose (Gy) covering $95(\%)$ of the PTV2 & 15 & $\begin{array}{l}<48.46 \\
\text { UNACCEPTABLE }\end{array}$ & \multicolumn{3}{|l|}{$\begin{array}{l}48.461 \\
\text { ACCEPTABLE }\end{array}$} & \multicolumn{2}{|c|}{$\begin{array}{l}56.43 \\
\text { GOOD }\end{array}$} & $\begin{array}{l}59.4 \\
\text { IDEAL }\end{array}$ \\
\hline [04] & Dose (Gy) covering $95(\%)$ of the PTV3 & 15 & $\begin{array}{l}<48.6 \\
\text { UNACCEPTABLE }\end{array}$ & \multicolumn{4}{|l|}{$\begin{array}{l}48.601 \\
\text { ACCEPTABLE }\end{array}$} & $\begin{array}{l}52.92 \\
\text { GOOD }\end{array}$ & $\begin{array}{l}54 \\
\text { IDEAL }\end{array}$ \\
\hline [05] & Volume (\%) of the PTV3-PTV2 covered by 56.7 (Gy) & 8 & $\begin{array}{l}>50 \\
\text { UNACCEPTABLE }\end{array}$ & $\begin{array}{l}50 \\
\text { MARGINAL }\end{array}$ & \multicolumn{2}{|c|}{$\begin{array}{l}40 \\
\text { ACCEPTABLE }\end{array}$} & & $\begin{array}{l}30 \\
\text { GOOD }\end{array}$ & \begin{tabular}{|l}
25 \\
IDEAL
\end{tabular} \\
\hline [06] & Conformation Number [51.3 (Gy), PTV3] & 10 & $\begin{array}{l}<0.5 \\
\text { UNACCEPTABLE }\end{array}$ & $\begin{array}{l}0.5 \\
\text { MARGINAL }\end{array}$ & \multicolumn{2}{|l|}{$\begin{array}{l}0.6 \\
\text { ACCEPTABLE }\end{array}$} & \multicolumn{2}{|l|}{$\begin{array}{l}0.7 \\
\text { GOOD }\end{array}$} & $\begin{array}{l}0.8 \\
\text { IDEAL }\end{array}$ \\
\hline [07] & Dose (Gy) covering $0.1(\mathrm{cc})$ of the SPINAL_CANAL [CRITICAL] & 10 & $\begin{array}{l}>55 \\
\text { UNACCEPTABLE }\end{array}$ & \multicolumn{2}{|l|}{$\begin{array}{l}54.99 \\
\text { ACCEPTABLE }\end{array}$} & \multicolumn{3}{|l|}{$\begin{array}{l}50 \\
\text { GOOD }\end{array}$} & $\begin{array}{l}45 \\
\text { IDEAL }\end{array}$ \\
\hline & Mean dose (Gy) to the PAROTID_L $\quad$ [CRITICAL] & 15 & $\begin{array}{l}>26 \\
\text { UNACCEPTABLE }\end{array}$ & $\begin{array}{l}26 \\
\text { MARGINAL }\end{array}$ & \multicolumn{2}{|l|}{$\begin{array}{l}25 \\
\text { ACCEPTABLE }\end{array}$} & \multicolumn{2}{|l|}{$\begin{array}{l}24 \\
\text { GOOD }\end{array}$} & $\begin{array}{l}23 \\
\text { IDEAL }\end{array}$ \\
\hline [09] & Mean dose (Gy) to the PAROTID_R & 5 & $\begin{array}{l}>38 \\
\text { UNACCEPTABLE }\end{array}$ & $\begin{array}{l}38 \\
\text { MARGINAL }\end{array}$ & \multicolumn{2}{|l|}{$\begin{array}{l}34 \\
\text { ACCEPTABLE }\end{array}$} & \multicolumn{2}{|l|}{$\begin{array}{l}30 \\
\text { GOOD }\end{array}$} & $\begin{array}{l}26 \\
\text { IDEAL }\end{array}$ \\
\hline [10] & Dose $(G y)$ covering $0.1(\mathrm{cc})$ of the Brainstem [CRITICAL] & 5 & $\begin{array}{l}>54.001 \\
\text { FAlL }\end{array}$ & \multicolumn{5}{|l|}{$\begin{array}{l}54.001 \\
\text { LABEL NOT SET }\end{array}$} & \begin{tabular}{|l|}
54 \\
PASS
\end{tabular} \\
\hline [11] & Dose (Gy) covering $0.5(\mathrm{cc})$ of the BRACHIAL_PLEXUSL. [CRITICAL] & 5 & $\begin{array}{l}>54.001 \\
\text { FAlL }\end{array}$ & \multicolumn{5}{|l|}{$\begin{array}{l}54.001 \\
\text { LABEL NOT SET }\end{array}$} & $\begin{array}{l}54 \\
\text { PASS }\end{array}$ \\
\hline [12] & Dose (Gy) covering 0.1 (cc) of the Brain & 5 & $\begin{array}{l}>65.001 \\
\text { FAIL }\end{array}$ & \multicolumn{5}{|l|}{$\begin{array}{l}65.001 \\
\text { LABEL NOT SET }\end{array}$} & $\begin{array}{l}65 \\
\text { PASS }\end{array}$ \\
\hline [13] & Dose (Gy) covering 0.1 (cc) of the Esophagus & 5 & $\begin{array}{l}>50.001 \\
\text { FAlL }\end{array}$ & \multicolumn{5}{|l|}{$\begin{array}{l}50.001 \\
\text { LABEL NOT SET }\end{array}$} & $\begin{array}{l}50 \\
\text { PASS }\end{array}$ \\
\hline [14] & Mean dose (Gy) to the Glottis & 5 & $\begin{array}{l}>45.001 \\
\text { FAlL }\end{array}$ & \multicolumn{5}{|l|}{$\begin{array}{l}45.001 \\
\text { LABEL NOT SET }\end{array}$} & $\begin{array}{l}45 \\
\text { PASS }\end{array}$ \\
\hline [15] & Dose (Gy) covering $0.1(\mathrm{cc})$ of the Mandible & 7 & $\begin{array}{l}>70.0001 \\
\text { FAlL }\end{array}$ & \multicolumn{5}{|l|}{$\begin{array}{l}70.0001 \\
\text { LABEL NOT SET }\end{array}$} & $\begin{array}{l}70 \\
\text { PASS }\end{array}$ \\
\hline [16] & Mean dose (Gy) to the OralCavity & 10 & $\begin{array}{l}>45 \\
\text { UNACCEPTABLE }\end{array}$ & \multicolumn{2}{|l|}{$\begin{array}{l}44.999 \\
\text { ACCEPTABLE }\end{array}$} & \multicolumn{3}{|l|}{$\begin{array}{l}40 \\
\text { GOOD }\end{array}$} & $\begin{array}{l}35 \\
\text { IDEAL }\end{array}$ \\
\hline [17] & Cumulative meterset over all treatment beams & -- & \multicolumn{7}{|c|}{ Unscored metric and/or performance bins are not defined. } \\
\hline [18] & Number of treatment beams & $\ldots$ & \multicolumn{7}{|c|}{ Unscored metric and/or performance bins are not defined. } \\
\hline [19] & Number of unique isocenters & $\ldots$ & \multicolumn{7}{|c|}{ Unscored metric and/or performance bins are not defined. } \\
\hline
\end{tabular}

Fig. 2 Scoring criteria as displayed on the ProKnow website created by ViewRay (Oakwood Village, OH, USA). PTV1 high-risk volume, PTV2 intermediate-risk volume, PTV3 low-risk volume, PTV planning target volume

MRI in the TPS using deformable image registration with default settings. The electron density of this deformed CT image was then used for dose calculation of all submitted plans. Once a plan had been created, participants were able to upload this plan onto the ProKnow platform. Afterwards, the plans were analyzed based on a scoring scheme created in conjunction with ViewRay. Each plan was then awarded a score out of 150 points. The delivery time (gantry and multileaf collimator [MLC] and beam-on time) of the plan had to be $20 \mathrm{~min}$ or less according to the treatment planning system, and the plans were to be calculated with the beam model of the local department of the planner.

\section{Challenge criteria and parameters}

The scoring criteria for this challenge were based on conformity of the prescription doses, sparing of OARs, and coverage of PTVs. One of the evaluated parameters was the conformation number $(\mathrm{CN})$, defined as TVRI*(TVRI)/(TV*VRI), with TVRI being the target volume (in this study PTV3) covered by the reference dose (cc), TV the total target volume (cc), and VRI the volume of the reference dose (cc). In total, 16 dose metrics were evaluated and are displayed in Fig. 2. For some metrics a range of points were available, depending on whether the dose achieved was deemed unacceptable, marginal, acceptable, good, or ideal in relation to what would be clinically acceptable. Other objectives for critical OARs were binary with pass or fail, scoring 0 points for any criteria not achieved (see Fig. 2 for scoring criteria). The sum of scores on all dose parameters results in a final score called the plan quality metric (PQM). Plans were defined as unacceptable if the total treatment time was longer than 20 min or if the PTV or OAR dose did not meet the constraints. 14 submissions were received and uploaded to this platform for analysis. Planners were also asked a few short questions about the amount of planning experience they had in general, and with the MRIdian system.

\section{Statistical analysis}

All dose metrics have a threshold value that results in maximum points. For all dose parameters, the relative deviation from the maximum points threshold values were calculated. 
Table 1 Contains the descriptive statistics of all the metrics evaluated in this study

\begin{tabular}{|c|c|c|c|c|}
\hline & Mean \pm SD & Median [range] & Q0.25 & Q0.75 \\
\hline$\overline{\text { PTV1 D95\% [Gy] }}$ & $67.5 \pm 1.2$ & $67.0[66.2-69.7]$ & 66.7 & 68.5 \\
\hline PTV1 D $0.1 \mathrm{cc}[\mathrm{Gy}]$ & $74.1 \pm 1.3$ & $73.9[72.0-76.6]$ & 73.5 & 74.3 \\
\hline PTV2 D95\% [Gy] & $59.0 \pm 1.0$ & $58.9[57.5-60.8]$ & 58.3 & 59.9 \\
\hline PTV3 D95\% $[\mathrm{Gy}]$ & $52.4 \pm 0.8$ & $52.6[50.7-53.5]$ & 52.3 & 52.9 \\
\hline PTV3-PTV2 V 56.7 Gy $[\%]$ & $22.3 \pm 7.8$ & $21.6[8.3-35.8]$ & 20.1 & 24.9 \\
\hline PTV3 conformation & $0.7 \pm 0.0$ & $0.7[0.7-0.8]$ & 0.7 & 0.7 \\
\hline Spinal canal $\mathrm{D}_{0.1 \mathrm{cc}}[\mathrm{Gy}]$ & $44.0 \pm 2.4$ & $43.4[39.0-48.8]$ & 42.7 & 45.6 \\
\hline Parotid gland left $\mathrm{D}_{\text {mean }}[\mathrm{Gy}]$ & $22.0 \pm 1.5$ & $22.3[17.8-23.8]$ & 21.6 & 22.8 \\
\hline Parotid gland right $\mathrm{D}_{\text {mean }}[\mathrm{Gy}]$ & $26.4 \pm 1.4$ & $26.0[24.4-29.1]$ & 25.6 & 27.0 \\
\hline Brainstem $\mathrm{D}_{0.1 \mathrm{cc}}[\mathrm{Gy}]$ & $42.1 \pm 4.0$ & $42.1[35.7-48.9]$ & 39.2 & 43.8 \\
\hline Brachial Plexus $\mathrm{D}_{0.5 \mathrm{cc}}[\mathrm{Gy}]$ & $51.2 \pm 1.6$ & $51.6[48.5-53.6]$ & 49.9 & 52.2 \\
\hline Brain $D_{0.1 c c}[G y]$ & $48.5 \pm 2.9$ & $49.7[43.2-52.4]$ & 46.5 & 50.6 \\
\hline Esophagus $\mathrm{D}_{0.1 \mathrm{cc}}[\mathrm{Gy}]$ & $44.5 \pm 2.7$ & $44.4[39.7-49.1]$ & 42.8 & 46.4 \\
\hline Glottis Dmean $[\mathrm{Gy}]$ & $42.3 \pm 1.9$ & $42.9[39.1-44.9]$ & 41.2 & 43.4 \\
\hline Mandible $\mathrm{D}_{0.1 \mathrm{cc}}[\mathrm{Gy}]$ & $59.7 \pm 2.4$ & $59.5[56.9-64.8]$ & 57.6 & 61.1 \\
\hline Oral cavity $\mathrm{D}_{\text {mean }}[\mathrm{Gy}]$ & $36.6 \pm 3.5$ & 36.0 [32.6-44.9] & 34.1 & 39.2 \\
\hline Plan quality metric (max 150$)$ & $140.1 \pm 6.5$ & $141.5[127.0-148.0]$ & 138.5 & 143.8 \\
\hline OAR points $(\max 72)$ & $70.3 \pm 1.8$ & $70.4[67.5-72.0]$ & 69.4 & 72.0 \\
\hline PTV points (max 78) & $69.7 \pm 5.5$ & $71.4[57.3-76.0]$ & 66.7 & 73.4 \\
\hline Number of beams & $21.1 \pm 13.5$ & $17.5[11.0-60.0]$ & 13.3 & 22.5 \\
\hline Number of MU & $918.9 \pm 232.7$ & 909.5 [539.0-1474.0] & 782.3 & 990.0 \\
\hline Number of segments & $147.6 \pm 24.2$ & $139.0[114.0-208.0]$ & 131.8 & 163.0 \\
\hline Beam-on time $[\mathrm{min}]$ & $1.5 \pm 0.4$ & $1.5[0.8-2.5]$ & 1.3 & 1.6 \\
\hline Total delivery time [min] & $18.7 \pm 1.7$ & $18.8[14.9-22.4]$ & 17.8 & 19.6 \\
\hline Planning experience [years] & $9.2 \pm 6.9$ & $7.0[2.0-20.0]$ & 3.1 & 14.3 \\
\hline ViewRay planning experience [years] & $1.1 \pm 0.6$ & $1.0[0.0-2.0]$ & 0.6 & 1.4 \\
\hline
\end{tabular}

$Q 0.25$ first quartile, $Q 0.75$ third quartile, $O A R$ organ at risk, $P T V$ planning target volume, $P T V 1$ high risk volume, $P T V 2$ intermediate risk volume, $P T V 3$ low risk volume, $M U$ monitor units, Dmean mean dose, min time in minutes, $D x$ dose recieved by respective volume, $V x$ percentage volume receiving $x \mathrm{~Gy}$

Differences with respect to the threshold were assessed with Wilcoxon signed-rank test. Besides dose metrics, beam-on time, treatment delivery time, number of beams, number of segments, number of monitor units (MU), and (ViewRay) planning experience were reported. Spearman's $\rho$ correlation coefficient was calculated to evaluate the correlation between each of the metrics. The total points for only PTV metrics and only OAR metrics were also included, to assess whether overall performance on PTV criteria corresponded to performance on OAR sparing. All statistics were performed in R (version 3.6.2, R Core Team 2020, Vienna, Austria), using the functions wilcox.test of the stats package and rcorr of the Hmisc package. A correlation coefficient above 0.7 was considered a strong correlation, above 0.5 was considered a moderate correlation, and below 0.5 was considered a weak correlation. $P$-values below 0.05 were considered significant.

\section{Results}

The detailed results for the 14 planners are included in Table 1 and Fig. 3. Each planner was able to achieve all the required constraints except for one planner for whom the total treatment delivery time exceeded the allowed time by $2.4 \mathrm{~min}$ (see Fig. 3). The median (range) number of beams used was 17.5 (11-60) and the median number of segments 139 (114-208), resulting in a total number of 909.5 MUs (539-1474), beam-on time of $1.5 \mathrm{~min}(0.8-2.5)$, and median total delivery time of $18.8 \mathrm{~min}$ (14.9-22.4). The median (range) IMRT planning experience for HNC of all users amounted to 7 years (2-20) and the ViewRay planning experience 1 year $(0-2)$.

The boxplot in Fig. 4 represents the relative deviation of the dose parameters with respect to the thresholds that result in maximum points for that metric. Note that the boxplot represents only 'higher' or 'lower,' which, depending on the metric, corresponds to either 'better' or 'worse.' The parameters for which the median performance of all centers was better than the threshold for maximum score are indicated 


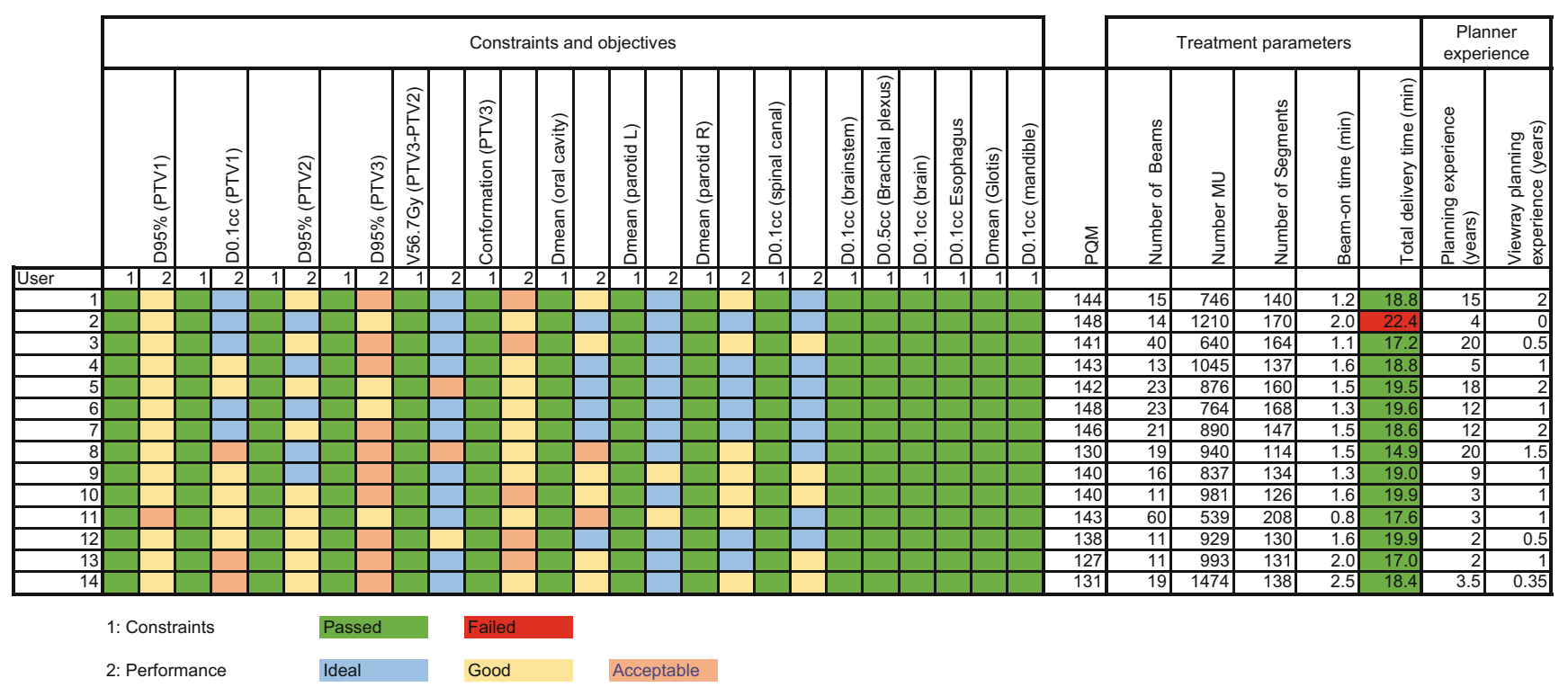

Fig. 3 Descriptive statistics of all metrics evaluated in this study. PTV1 high-risk volume, PTV2 intermediate-risk volume, PTV3 low-risk volume, PTV planning target volume

with a plus sign in the boxplot, whereas a minus sign was used to indicate the opposite. The PTV1-D $95 \%$, PTV1-D $0.1 \mathrm{cc}$, PTV2-D $95 \%$, and PTV3-D $95 \%$ all deviate less than $7 \%$ from the optimal. For all OAR dose parameters, except the $\mathrm{D}_{\text {mean }}$ of the right parotid gland and the $\mathrm{D}_{\text {mean }}$ of the oral cavity, the median score of all centers was better than the threshold that results in maximum score. Six centers achieved the maximum number of points for the OAR dose parameters. None of the centers had an unacceptable performance on any of the metrics.

Fig. 5 shows the correlation matrix for all metrics evaluated in this study. Only correlation coefficients considered as moderate (i.e., lower than -0.5 or higher than 0.5 ) are displayed in the matrix. Significance levels are indicated as estimated by the rcorr function in R. The number of beams is moderately correlated to the number of MUs $(p=0.015)$, the number of segments $(p=0.0081)$, and the beam-on time $(p=0.0087)$. The number of MUs is also strongly correlated to the beam-on time $(p<0.0001)$. No correlation was observed between the number of beams and any of the dose metrics. The number of segments is strongly correlated to the brainstem- $\mathrm{D}_{0.1 c c}(p=0.0022)$, and moderately correlated to the PTV1- $\mathrm{D}_{0.1 c c}(p=0.025)$. The total delivery time is moderately correlated to two dose metrics: PTV3-D $\mathrm{D}_{95 \%}$ $(p=0.0095)$ and $\mathrm{D}_{\text {mean }}$ of the oral cavity $(p=0.011)$. The points total on the PTV metrics is only moderately correlated to one OAR metric: brainstem- $\mathrm{D}_{0.1 \mathrm{cc}}(p=0.049)$. The points total on OAR metrics is only moderately correlated to one PTV metric: PTV3- $\mathrm{D}_{95} \%(p=0.047)$. The correlations between total OAR points and PTV metrics are shown in Fig. 6.

\section{Discussion}

In recent years there have been a few comparative multicenter planning studies, both for stereotactic body radiotherapy (SBRT) [15-18] and for IMRT, even for HNC [19]. These studies helped to share experiences for specific treatment indications, and in some cases even led to long-term plan improvement through targeted intervention, i.e., sharing the best plans regarding sparing OARs with the other centers [19]. However, to the best of our knowledge, this is the first study comprising ViewRay TPS for HNC. Planning HNC treatment on such platforms as the MRIdian system presents some technical difficulties compared to the current standard. In most high-volume centers, HNC treatments are planned with volumetric modulated arc therapy (VMAT), which allows the delivery of a highly conformal dose distribution in a very short timeframe $[20,21]$. The sole technique available with the MRIdian system is step-and-shoot IMRT. The delivery time is therefore much longer than on a standard linac (15-20 min), mainly due to the slow MLC motion and the gantry rotation speed. Additional limitations originate from limited beam angles (dead zone 30-33 degrees and recommended avoidance of couch edges), the lack of a collimator preventing the use of the MLC in different directions, and no option to use more efficient delivery techniques such as sliding window.

In the present planning study, we have demonstrated that treatment plans of high quality and complexity for treating $\mathrm{HNC}$ on the MRIdian platform resulting in reasonable treatment delivery time can be achieved with sufficient IMRT planning experience and through increasing the number of segments used for a particular plan. 
Fig. 4 Relative difference of all evaluated metrics with respect to the value that results in maximum score. The +'and signs on the right side indicate whether the median performance of all centers was better or worse than the ideal, respectively. Asterisks on the left indicate whether the scores were significantly different from the ideal score $\left({ }^{*} p<0.05, * * p<0.01\right.$, $* * * p<0.001) . P T V 1$ high-risk volume, $P T V 2$ intermediate-risk volume, $P T V 3$ low-risk volume, $P T V$ planning target volume

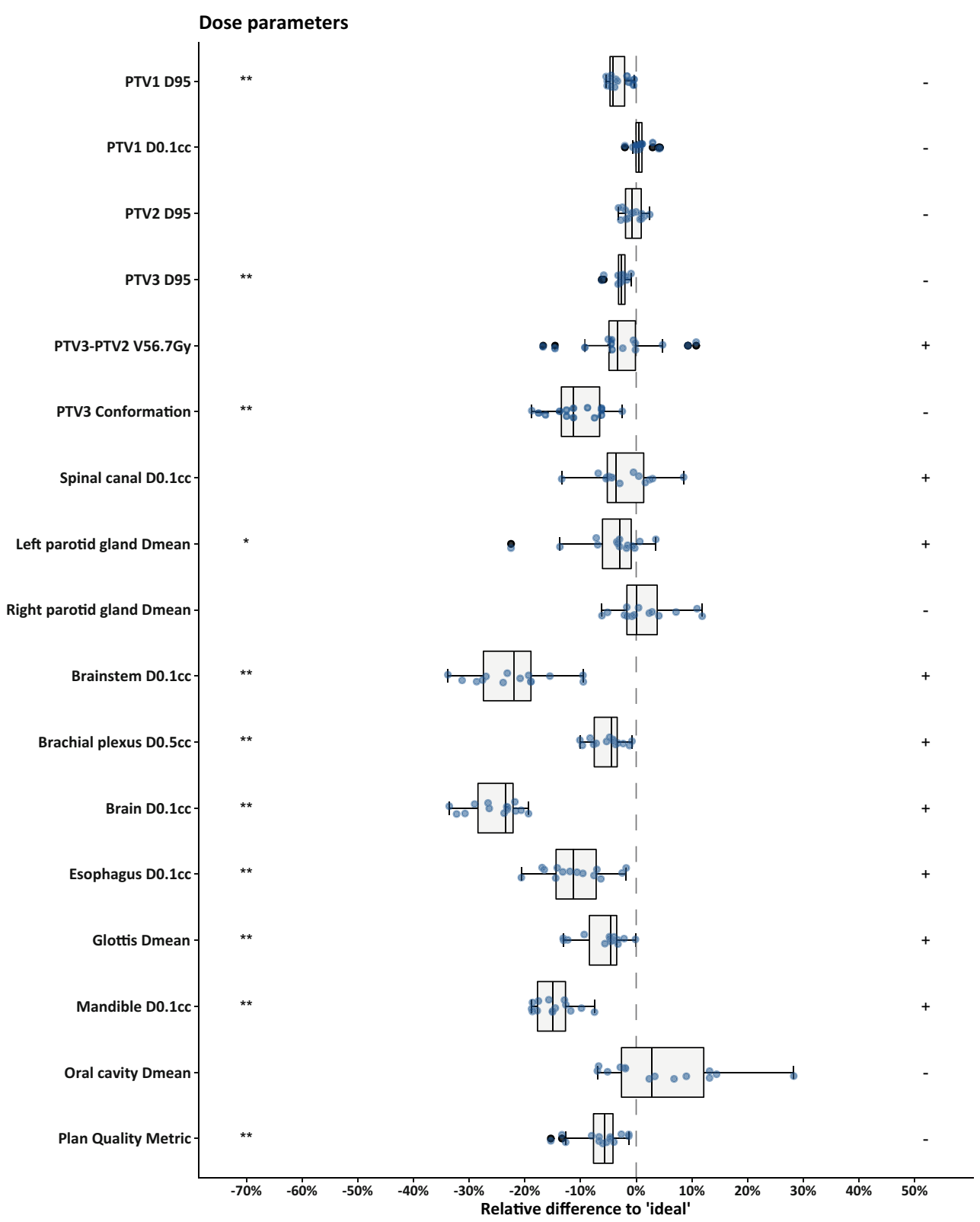

Recently developed hybrid machines consisting of a linear accelerator and integrated MRI (MR linacs) could allow a) better visualization of tumor and organs at risk, such as parotid glands during patient positioning and daily treatment; b) daily imaging without additional radiation exposure; c) reduction of established safety margins for the treatment volumes; and, finally, d) frequent adaptation of target volumes according to changes in patient weight and tumor anatomy during the radiotherapy course. These procedures would facilitate a high-precision treatment and help reduce dose exposure of critical structures. MR linacs are already in use in several centers worldwide and published studies have shown that the quality of MR linac plans achieved was sufficient and not inferior to other linacs for various anatomical sites and diseases [21-23]. Authors from dif- ferent institutions showed that MR-guided radiotherapy is tolerated by the patients [24], compared MR-based planning with conventional linac-based stereotactic radiotherapy [25], and discussed potential advantages [26]. These studies mainly reported the clinical feasibility of stereotactic body radiation therapy MR-guided radiotherapy, but data for complex and large target volumes such as used for curative, conventional fractionated therapy like head and neck treatments are still very rare, and all of the published studies for HNC have been conducted either in non-linac (cobalt) platforms, or had no specific focus on plan optimization $[27,28]$. These first applications appear encouraging: Chen et al. have shown feasibility of MR-guided radiotherapy for both primary cases and in the challenging situation of re-irradiation of recurrent $\mathrm{HNC}[27,29]$. MR-adapted planning 


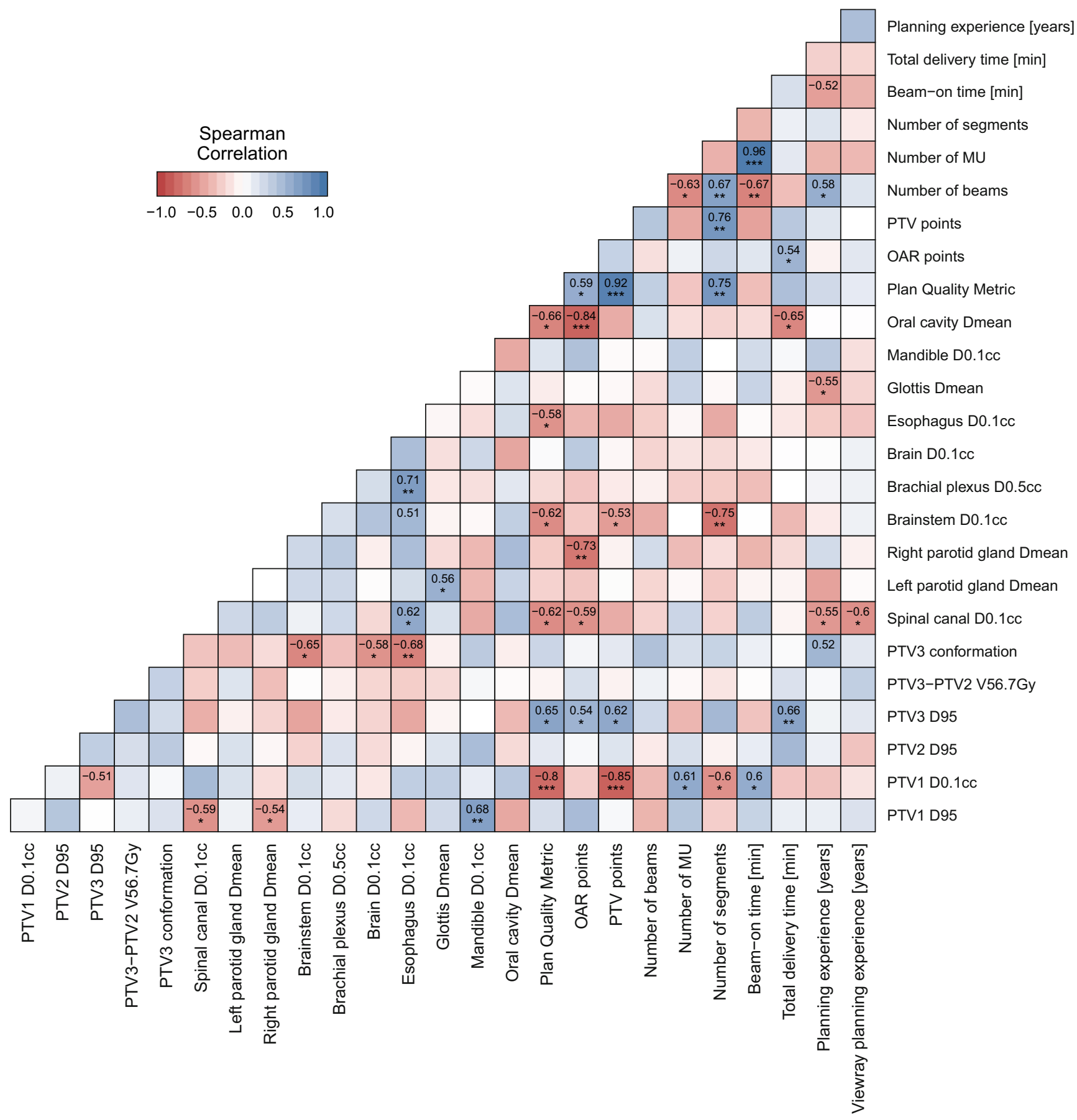

Fig. 5 Correlation matrix representing Spearman's $\rho$ correlation coefficients for all evaluated metrics in this study. Only correlations that are considered moderate $(\varrho<-0.5$ or $\varrho>0.5)$ are displayed. Asterisks indicate significance $(* p<0.05, * * p<0.01, * * * p<0.001)$. OAR organ at risk, $P T V$ planning target volume, $M U$ monitor units, PTV1 high-risk volume, $P T V 2$ intermediate-risk volume, $P T V 3$ low-risk volume

could allow for repetitive and more precise plan adaptations, taking into account such challenges as weight loss, interfractional differences, and organ and tumor motion [30-32], which are all important problems of head and neck radiotherapy. Advantages in this regard might allow for safer and personalized radiotherapy in the future. The MRIdian ${ }^{\circledR}$ technology, which was used in this study, combines $0.35 \mathrm{~T}$
MRI with a multileaf-collimator linac and has already been described in detail before [9, 33-35]. Furthermore, various methods and procedures for quality assurance of the system are meanwhile also well established [31, 36-38].

The results of this study could demonstrate that IMRT plans of relatively high quality can already be developed after a median ViewRay user experience of about 1 year if 

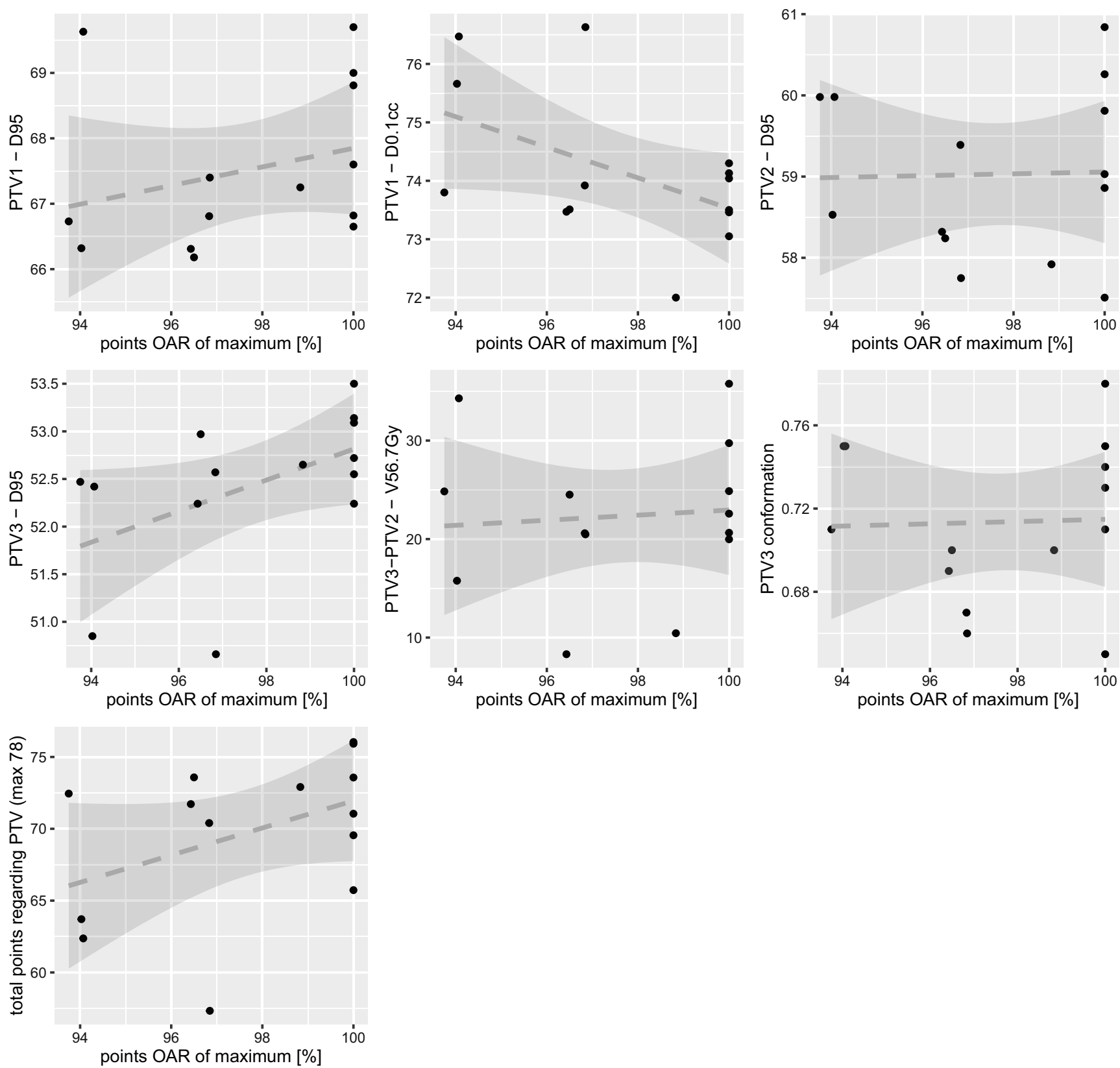

Fig. 6 Plots indicating the relationship between different PTV dose parameters (y-axis) versus the total number of points achieved for OAR dose parameters (x-axis). The dotted line represents the linear fit and the gray shaded areas represent $95 \%$ confidence intervals. OAR organ at risk, $P T V$ planning target volume, PTV1 high-risk volume, PTV2 intermediate-risk volume, PTV3 low-risk volume

the planner has gained sufficient experience with IMRT for $\mathrm{HNC}$ in the past (in the present study a median of 7 years). All of the plans developed would have been applicable and acceptable in clinical routine without any compromises in PTV or OARs constraints. Interestingly, 6/14 centers could even achieve the maximal quality score possible for OAR sparing. None of the centers were able to achieve an ideal conformation number of 0.8 , but $10 / 14$ centers achieved good performance within the range $(0.7-0.8)$, whereas $4 / 14$ centers achieved acceptable performance within range (0.6-0.7). Although none of the plans had a marginal or unacceptable conformation number, the moderate, negative correlation between conformation number and several OARs shows the challenge of target coverage and simultaneous OAR sparing. Other interesting correlations between dose parameters and plan characteristics were observed in this study (Fig. 5). Planning experience was moderately correlated with improved planning results for some specific OARs like spinal cord $\mathrm{D}_{0.1 \mathrm{cc}}(\rho=-0.55)$ or glottis $\mathrm{D}_{\text {mean }}(\rho=-0.55)$. In line with this observation, previous studies have demonstrated improved outcomes with increasing planning experience for patients treated with 
IMRT for HNCs [39, 40], and other malignancies [41]. Intriguingly, the planning experience did not correlate with the overall points scored in the present challenge. A possible explanation for this phenomenon could be that all of the participants of this study originated from high-volume centers, where the number of patients treated per time unit and the resulting experience are already high [40]. Another interesting observation of the present study was the significant correlation of the number of segments used and both the "PTV points" $(\rho=0.76)$ and the PQM $(\rho=0.75)$, making this the most important factor for plan quality. Although a higher number of segments could generally correlate with longer delivery times as has been shown before [42], this was not the case with the present example. The relatively high number of beams used by all of the participants (median 17.5) resulting in long delivery times could explain the lack of significant association of segments and delivery duration. However, a slight reduction of the segments and increase of beam angles might allow for faster treatment in future planning [42]. Interestingly, increasing the number of beams was associated with an increased segment number $(\rho=0.67)$, even though the number of total segments can be limited in the planning system. Sutton et al. recommends not more than 3-6 segments per beam for good plan quality of IMRT plans [43], which is clearly less than the numbers used here (median 139 segments for 17.5 beams). The treatment delivery time remains an issue of step-and-shoot IMRT and of the present platform: with a median of $18.8 \mathrm{~min}$, it is longer compared to other modern IMRT and especially VMAT platforms [20]. This was the only parameter not met by $100 \%$ of the participants. As the median beam-on time of $1.5 \mathrm{~min}$ for a median of 909 MUs was comparable or even better than Halcyon and True-Beam plans [44], the slower application is mainly attributed to the MLC parameters such as leaf speed. An increase in the number of beams was actually associated with a reduction in the total monitor units $(\rho=-0.63)$, and thus with a reduced total delivery time. An upgrade with faster MLC movement would surely facilitate wider implementation of the platform in clinical routine. Patients with $\mathrm{HNC}$ in advanced stages, with rapidly reacting tumors like human papilloma virus-positive oropharyngeal or EpsteinBarr Virus-positive nasopharyngeal carcinoma, with irradiation of both neck sides, with the PTV in close proximity to various OARs, and with moving targets such as small laryngeal carcinomas could possibly benefit the most from treatment on MR linac platforms due to the possibility of online imaging during treatment and repeated adaptations. However, intrafractional and interfractional variations for this cancer type do not occur as frequently and unexpectedly as in moving abdominal organs or the pelvic region. Furthermore, only conventional fractionation or generally low-dose-per-fraction regimens are mostly used for this indication. The potential added value of online adaptation should be considered and carefully weighed against a prolonged treatment time. An offline and less frequent than daily adaptation could be sufficient for most HNC cases. Taken together, the planning time is not as crucial for this indication as, in contrast to SBRT applications, planning will be mostly done offline. Much more important for applying a standard regimen of radiotherapy for HNC over 7 weeks and over 30 fractions will be the treatment and beam delivery time. Future improvements in this direction, like integrating sliding window and VMAT techniques in the MR linac, will surely improve patient comfort and compliance. Due to this reason, we decided to assess these last parameters in this study.

There are a few limitations of this study. First or all, the present analysis does not focus on a clinically challenging situation, e.g., SBRT for spinal metastases [15], nor on implementation of society guidelines [16], nor on optimizing OAR sparing [19]. The focus of the present work was to investigate the application and the features of the modern technology of MR linac and a relatively novel planning system for the new indication of head and neck cancer and to generate hypotheses for future plan optimization. The number of centers that participated is probably enough for such purposes, as in the Dutch planning study for HNC a similar number of centers (15) was enough to be even practice changing [19].

Another possible shortcoming of this multicenter study could be the omission of calculating a complexity index (CI) for all plans as described by several authors before for planning studies of IMRT or SBRT [45]. However, Hernandez et al. stressed the importance of CI implementation, especially for comparison of multicenter results when using different planning systems, as the differences when comparing other quality metrics for plans developed in the same planning system were not so pronounced [46]. Since all the participants in this study used the same planning system, the complexity index was not evaluated. Also, as only one case was included, the results might not be applicable to all HNC plans.

Furthermore, prior to actual treatment delivery, quality assurance (QA) is required in order to avoid dose delivery errors [47]. Nevertheless, the evaluation of in vivo dosimetry was not within the scope of this study, so no measurements of the actual plans were performed.

Despite these limitations, this is the first study providing treatment planning recommendations for treating $\mathrm{HNC}$ on the MRIdian platform as a result of a multicentric effort of experienced users. In addition, this is one of the first studies demonstrating feasibility and quality of planning for HNC on the MRidian platform and providing recommendations for the numbers of beams and segments to start with. 


\section{Conclusion}

Clinically acceptable and excellent treatment plans for $\mathrm{HNC}$ were achieved by most users on the MRIdian platform. The quality of these plans can be optimized through implementation of a higher number of segments and increasing experience of the planner and can thereby achieve clinically acceptable results. Inauguration of MR linacs for routine clinical treatment of HNC patients appears already feasible.

Supplementary Information The online version of this article (https:// doi.org/10.1007/s00066-021-01771-8) contains supplementary material, which is available to authorized users.

Funding Open Access funding provided by Universität Zürich.

\section{Declarations}

Conflict of interest M. Chamberlain, J. Krayenbuehl, J. E. van Timmeren, L. Wilke, N. Andratschke, H. Garcia Schüler, S. Tanadini-Lang, M. Guckenberger, and P. Balermpas declare that they have no competing interests.

Ethical standards For this article no studies with human participants or animals were performed by any of the authors. As the planning study did not involve any patients or animals, ethical vote was waived. The patient signed informed consent for use of data and images.

Open Access This article is licensed under a Creative Commons Attribution 4.0 International License, which permits use, sharing, adaptation, distribution and reproduction in any medium or format, as long as you give appropriate credit to the original author(s) and the source, provide a link to the Creative Commons licence, and indicate if changes were made. The images or other third party material in this article are included in the article's Creative Commons licence, unless indicated otherwise in a credit line to the material. If material is not included in the article's Creative Commons licence and your intended use is not permitted by statutory regulation or exceeds the permitted use, you will need to obtain permission directly from the copyright holder. To view a copy of this licence, visit http://creativecommons.org/licenses/by/4. $0 /$.

\section{References}

1. Leitzen C, Wilhelm-Buchstab T, Müdder T et al (2018) Patient positioning in head and neck cancer: setup variations and safety margins in helical tomotherapy. Strahlenther Onkol 194:386-391. https://doi.org/10.1007/s00066-018-1265-7

2. Qi XS, Hu AY, Lee SP et al (2013) Assessment of interfraction patient setup for head-and-neck cancer intensity modulated radiation therapy using multiple computed tomography-based image guidance. Int J Radiat Oncol Biol Phys 86:432-439. https://doi.org/10. 1016/j.ijrobp.2013.01.022

3. Stoiber EM, Bougatf N, Teske H et al (2017) Analyzing human decisions in IGRT of head-and-neck cancer patients to teach image registration algorithms what experts know. Radiat Oncol 12:104. https://doi.org/10.1186/s13014-017-0842-8

4. Bell K, Licht N, Rübe C, Dzierma Y (2018) Image guidance and positioning accuracy in clinical practice: influence of positioning errors and imaging dose on the real dose distribution for head and neck cancer treatment. Radiat Oncol 13:190. https://doi.org/10. 1186/s13014-018-1141-8

5. Heukelom J, Fuller CD (2019) Head and neck cancer adaptive radiation therapy (ART): conceptual considerations for the informed clinician. Semin Radiat Oncol 29:258-273. https://doi.org/10.1016/ j.semradonc.2019.02.008

6. Nutting CM, Morden JP, Harrington KJ et al (2011) Parotid-sparing intensity modulated versus conventional radiotherapy in head and neck cancer (PARSPORT): a phase 3 multicentre randomised controlled trial. Lancet Oncol 12:127-136. https://doi.org/10.1016/ S1470-2045(10)70290-4

7. Hvid CA, Elstrøm UV, Jensen K, Grau C (2018) Cone-beam computed tomography (CBCT) for adaptive image guided head and neck radiation therapy. Acta Oncol 57:552-556. https://doi.org/10. 1080/0284186X.2017.1398414

8. Spezi E, Downes P, Jarvis R et al (2012) Patient-specific three-dimensional concomitant dose from cone beam computed tomography exposure in image-guided radiotherapy. Int J Radiat Oncol Biol Phys 83:419-426. https://doi.org/10.1016/j.ijrobp.2011.06.1972

9. Mutic S, Dempsey JF (2014) The ViewRay system: magnetic resonance-guided and controlled radiotherapy. Semin Radiat Oncol 24:196-199. https://doi.org/10.1016/j.semradonc.2014.02.008

10. Biau J, Lapeyre M, Troussier I et al (2019) Selection of lymph node target volumes for definitive head and neck radiation therapy: a 2019 Update. Radiother Oncol 134:1-9. https://doi.org/10.1016/ j.radonc.2019.01.018

11. Grégoire V, Evans M, Le Q-T et al (2018) Delineation of the primary tumour Clinical Target Volumes (CTV-P) in laryngeal, hypopharyngeal, oropharyngeal and oral cavity squamous cell carcinoma: AIRO, CACA, DAHANCA, EORTC, GEORCC, GORTEC, HKNPCSG, HNCIG, IAG-KHT, LPRHHT, NCIC CTG, NCRI, NRG Oncology, PHNS, SBRT, SOMERA, SRO, SSHNO, TROG consensus guidelines. Radiother Oncol 126:3-24. https://doi.org/ 10.1016/j.radonc.2017.10.016

12. Navran A, Heemsbergen W, Janssen T, Hamming-Vrieze O, Jonker M, Zuur C, Verheij M, Remeijer P, Sonke J-J, van den Brekel M, Al-Mamgani A (2019) The impact of margin reduction on outcome and toxicity in head and neck cancer patients treated with imageguided volumetric modulated arc therapy (VMAT). Radiotherapy and Oncology 130:25-31

13. Brouwer CL, Steenbakkers RJHM, Bourhis J, Budach W, Grau C, Grégoire V, van Herk M, Lee A, Maingon P, Nutting C, O'Sullivan B, Porceddu SV, Rosenthal DI, Sijtsema NM, Langendijk JA (2015) CT-based delineation of organs at risk in the head and neck region: DAHANCA, EORTC, GORTEC, HKNPCSG, NCIC CTG, NCRI, NRG Oncology and TROG consensus guidelines. Radiotherapy and Oncology 117(1):83-90

14. Klüter S (2019) Technical design and concept of a 0.35 T MRLinac. Clin Transl Radiat Oncol 18:98-101. https://doi.org/10. 1016/j.ctro.2019.04.007

15. Esposito M, Masi L, Zani M et al (2019) SBRT planning for spinal metastasis: indications from a large multicentric study. Strahlenther Onkol 195:226-235. https://doi.org/10.1007/s00066-018-1383-2

16. Moustakis C, Blanck O, Ebrahimi Tazehmahalleh F et al (2017) Planning benchmark study for SBRT of early stage NSCLC: results of the DEGRO working group stereotactic radiotherapy. Strahlenther Onkol 193:780-790. https://doi.org/10.1007/s00066017-1151-8

17. Moustakis C, Chan MKH, Kim J et al (2018) Treatment planning for spinal radiosurgery: a competitive multiplatform benchmark challenge. Strahlenther Onkol 194:843-854. https://doi.org/10. 1007/s00066-018-1314-2

18. Giglioli FR, Garibaldi C, Blanck O et al (2020) Dosimetric multicenter planning comparison studies for stereotactic body radiation therapy: methodology and future perspectives. Int J Radiat On- 
col Biol Phys 106:403-412. https://doi.org/10.1016/j.ijrobp.2019. 10.041

19. Verbakel WFAR, Doornaert PAH, Raaijmakers CPJ et al (2019) Targeted intervention to improve the quality of head and neck radiation therapy treatment planning in the Netherlands: short and longterm impact. Int J Radiat Oncol Biol Phys 105:514-524. https://doi. org/10.1016/j.ijrobp.2019.07.005

20. Studenski MT, Bar-Ad V, Siglin J et al (2013) Clinical experience transitioning from IMRT to VMAT for head and neck cancer. Med Dosim 38:171-175. https://doi.org/10.1016/j.meddos.2012.10.009

21. Menten MJ, Fast MF, Nill S et al (2016) Lung stereotactic body radiotherapy with an MR-linac-quantifying the impact of the magnetic field and real-time tumor tracking. Radiother Oncol 119:461-466. https://doi.org/10.1016/j.radonc.2016.04.019

22. Saenz DL, Paliwal BR, Bayouth JE (2014) A dose homogeneity and conformity evaluation between ViewRay and pinnacle-based linear accelerator IMRT treatment plans. J Med Phys 39:64-70. https:// doi.org/10.4103/0971-6203.131277

23. van de Schoot AJAJ, van den Wollenberg W, Carbaat C et al (2019) Evaluation of plan quality in radiotherapy planning with an MRlinac. Phys Imaging Radiat Oncol 10:19-24. https://doi.org/10. 1016/j.phro.2019.04.004

24. Tetar S, Bruynzeel A, Bakker R et al (2018) Patient-reported outcome measurements on the tolerance of magnetic resonance imaging-guided radiation therapy. Cureus 10:e2236. https://doi.org/10. 7759/cureus.2236

25. Merna C, Rwigema J-CM, Cao M et al (2016) A treatment planning comparison between modulated tri-cobalt-60 teletherapy and linear accelerator-based stereotactic body radiotherapy for central earlystage non-small cell lung cancer. Med Dosim 41:87-91. https://doi. org/10.1016/j.meddos.2015.09.002

26. Henke L, Kashani R, Yang D et al (2016) Simulated online adaptive magnetic resonance-guided stereotactic body radiation therapy for the treatment of oligometastatic disease of the abdomen and central thorax: characterization of potential advantages. Int J Radiat Oncol Biol Phys 96:1078-1086. https://doi.org/10.1016/j.ijrobp.2016.08. 036

27. Chen AM, Cao M, Hsu S et al (2017) Magnetic resonance imaging guided reirradiation of recurrent and second primary head and neck cancer. Adv Radiat Oncol 2:167-175. https://doi.org/10.1016/ j.adro.2017.02.002

28. Raghavan G, Kishan AU, Cao M, Chen AM (2016) Anatomic and dosimetric changes in patients with head and neck cancer treated with an integrated MRI-tri-(60)Co teletherapy device. Br J Radiol 89:20160624. https://doi.org/10.1259/bjr.20160624

29. Chen AM, Hsu S, Lamb J et al (2018) MRI-guided radiotherapy for head and neck cancer: initial clinical experience. Clin Transl Oncol 20:160-168. https://doi.org/10.1007/s12094-017-1704-4

30. Levin-Epstein R, Cao M, Lee P et al (2018) Magnetic resonanceguided inter-fraction monitoring opens doors to delivering safer reirradiation: an illustrative case report and discussion. Cureus 10:e2479. https://doi.org/10.7759/cureus.2479

31. Li HH, Rodriguez VL, Green OL et al (2015) Patient-specific quality assurance for the delivery of (60)Co intensity modulated radiation therapy subject to a 0.35 - $\mathrm{T}$ lateral magnetic field. Int $\mathrm{J}$ Radiat Oncol Biol Phys 91:65-72. https://doi.org/10.1016/j.ijrobp. 2014.09.008

32. Thomas DH, Santhanam A, Kishan AU et al (2018) Initial clinical observations of intra- and interfractional motion variation in. $\mathrm{Br} \mathrm{J}$ Radiol 91:20170522. https://doi.org/10.1259/bjr.20170522
33. Cai B, Li H, Yang D et al (2017) Performance of a multi leaf collimator system for MR-guided radiation therapy. Med Phys 44:6504-6514. https://doi.org/10.1002/mp.12571

34. Ginn JS, Agazaryan N, Cao M et al (2017) Characterization of spatial distortion in a $0.35 \mathrm{~T}$ MRI-guided radiotherapy system. Phys Med Biol 62:4525-4540. https://doi.org/10.1088/1361-6560/ aa6ela

35. Hu Y, Rankine L, Green OL et al (2015) Characterization of the onboard imaging unit for the first clinical magnetic resonance image guided radiation therapy system. Med Phys 42:5828-5837. https:// doi.org/10.1118/1.4930249

36. Andreozzi JM, Mooney KE, Brůža P et al (2018) Remote Cherenkov imaging-based quality assurance of a magnetic resonance imageguided radiotherapy system. Med Phys 45:2647-2659. https://doi. org/10.1002/mp.12919

37. Mein S, Rankine L, Adamovics J et al (2017) Development of a 3D remote dosimetry protocol compatible with MRgIMRT. Med Phys 44:6018-6028. https://doi.org/10.1002/mp.12565

38. Wang Y, Wang M, Jiang J (2017) An analysis of intrinsic variations of low-frequency shear wave speed in a stochastic tissue model: the first application for staging liver fibrosis. Phys Med Biol 62:1149-1171. https://doi.org/10.1088/1361-6560/aa51ac

39. Jain S, Popple R, Szychowski J et al (2019) Radiation oncologist characteristics and their association with outcomes in patients with head and neck cancer. Pract Radiat Oncol 9:e322-e330. https://doi. org/10.1016/j.prro.2019.01.001

40. Boero IJ, Paravati AJ, Xu B et al (2016) Importance of radiation oncologist experience among patients with head-and-neck cancer treated with intensity-modulated radiation therapy. J Clin Oncol 34:684-690. https://doi.org/10.1200/JCO.2015.63.9898

41. Yang GQ, Mhaskar R, Rishi A et al (2018) Intensity-modulated radiotherapy at high-volume centers improves survival in patients with esophageal adenocarcinoma receiving trimodality therapy. Dis Esophagus. https://doi.org/10.1093/dote/doy 124

42. Bratengeier K, Gainey MB, Flentje M (2011) Fast IMRT by increasing the beam number and reducing the number of segments. Radiat Oncol 6:170. https://doi.org/10.1186/1748-717X-6-170

43. Sutton J, Kabiru D, Neu M et al (2012) Define baseline levels of segments per beam for intensity-modulated radiation therapy delivery for brain, head and neck, thoracic, abdominal, and prostate applications. Med Dosim 37:15-19. https://doi.org/10.1016/j.meddos. 2010.12.006

44. Li T, Scheuermann R, Lin A et al (2018) Impact of multi-leaf collimator parameters on head and neck plan quality and delivery: a comparison between halcyon ${ }^{\mathrm{TM}}$ and Truebeam ${ }^{\circledR}$ treatment delivery systems. Cureus 10:e3648. https://doi.org/10.7759/cureus.3648

45. Villaggi E, Hernandez V, Fusella M et al (2019) Plan quality improvement by DVH sharing and planner's experience: results of a SBRT multicentric planning study on prostate. Phys Med 62:73-82. https://doi.org/10.1016/j.ejmp.2019.05.003

46. Hernandez V, Saez J, Pasler M et al (2018) Comparison of complexity metrics for multi-institutional evaluations of treatment plans in radiotherapy. Phys Imaging Radiat Oncol 5:37-43. https://doi.org/ 10.1016/j.phro.2018.02.002

47. Esposito M, Villaggi E, Bresciani S et al (2020) Estimating dose delivery accuracy in stereotactic body radiation therapy: A review of in-vivo measurement methods. Radiother Oncol 149:158-167. https://doi.org/10.1016/j.radonc.2020.05.014 